\title{
Calculation of X-ray spectra for a side window tube
}

\author{
M. Procop*, V.-D. Hodoroaba* and A. Bjeoumikhov** \\ * Bundesanstalt fuer Materialforschung und -pruefung (BAM), D-12200, Berlin, Germany \\ ** Institut fuer wissenschaftlichen Geraetebau (IfG), D-12849 Berlin, Germany
}

Micro-X-ray fluorescence ( $\mu$-XRF) is becoming a new analytical tool in combination with the scanning electron microscope (SEM) [1], [2]. Due to the much lower background in comparison with electron probe microanalysis the detection limits come in the order of some $10^{2} \mathrm{ppm}$ in case that an energy dispersive spectrometer is used, and even lower in case of a wavelength dispersive spectrometer. Small, low power X-ray tubes are available as attachments for the SEM. The diameter of the specimen area illuminated by the X-rays is about $1 \mathrm{~mm}^{2}$ in case of an aperture or ranges between 50 and $100 \mu \mathrm{m}^{2}$ in case of a focussing X-ray optics behind the tube.

For quantitative XRF the tube spectrum must be known. It has to be multiplied with the transmission of the X-ray optics in case of $\mu$-XRF to get the exciting spectrum. The iMOXS source manufactured by IfG operates with microfucus tube [3] having a side window and $12^{\circ}$ target inclination. In order to get the tube spectrum we measured X-ray spectra for the target materials of interest (Mo, Rh, W) in a SEM with an energy dispersive spectrometer of known spectrometer efficiency (counts per incident photons) [4] and with a carefully determined solid angle [5]. Measurements were carried out for perpendicular electron incidence, for a take-off angle (TOA) of $35^{\circ}$ and for high voltages ranging from 5 to $30 \mathrm{kV}$.

From the measured spectra the intensity of characteristic lines was found after subtraction of a physical background [6] and deconvolution of K-, L- and M-series. These net line intensities were divided by the spectrometer efficiency, the solid angle and the accumulated charge to get them as emitted photons per steradian per nC. Applying absorption correction one gets the X-ray yield $Y$. We applied the XPP absorption correction method [7].

It is well known that the X-ray yield follows for not too high overvoltage ratios an exponential law of the form

$$
Y=K\left(\frac{E}{E_{c}}-1\right)^{n}=K(U-1)^{n}
$$

The constants $K$ and $n$ have been determined in the past for a lot of elements by a $\log (Y)$ versus $\log (U-1)$ plot (s. [5], [8] and references therein), but not yet for the three metals Mo, Rh and W. Our results for these three metals are summarised in Table 1. $K$ is given there for the most intensive line coming from the ionised shell. $K$ 's for the weaker lines have to be calculated using the partial yields tabulated e.g. by Elam et al. [9].

The emitted characteristic spectrum for any primary electron energy $E$ covering the whole range of tube voltages, tilt angle $T A$ and take-off angle $T O A$ can now be calculated from

$$
I=Y(E) * A C(T O A) * R(T A) / R(0)
$$

$R$ means the backscattering correction factor, which was calculated as proposed in ref. [10]. Finally, the intensities have to be multiplied with the tube window absorption (e.g. $100 \mu \mathrm{m} \mathrm{Be}$ ) and the transmission of the X-ray optics to get the characteristic spectrum exciting the X-ray fluorescence. A bremsstrahlung background

$$
B=K_{B} \frac{E_{0}-E}{E^{m}}
$$


multiplied with the spectrometer efficiency was scaled to the spectra measured for perpendicular electron incidence and $\mathrm{TOA}=35^{\circ}$. Table 1 lists also these $K_{B}$ values. The background in case of the tilted target was calculated by multiplying $K_{B}$ with the ratio of backscattering factors for the actual $\mathrm{TW}$ and for $\mathrm{TW}=0^{\circ}$.

The given procedure has been checked in the SEM for tilted specimens and the detector in horizontal position (i.e. $\mathrm{TOA}=\mathrm{TA}$ ). With a few exceptions still under investigation the agreement between measured and calculated spectra is better than $5 \%$.

TABLE 1. Values of empirical constants in Eqs. (1) and (3)

\begin{tabular}{ccccc}
\hline Element & Shell & $\boldsymbol{K}$ (Photons/nC/msr) & $\boldsymbol{n}$ & $\boldsymbol{K}_{\boldsymbol{B}}$ (1/keV) \\
\hline Mo & K & 370 & 1.72 & $2.62 \mathrm{e}-006$ \\
& L1 & 10 & 1.40 & \\
& L2 & 47 & 1.40 & $2.48 \mathrm{e}-006$ \\
Rh & L3 & 115 & 1.40 & \\
& K & 353 & 1.7 & \\
& L1 & 7.0 & 1.40 & $2.44 \mathrm{e}-006$ \\
W2 & 52.5 & 1.40 & \\
& L3 & 129 & 1.40 & \\
& L1 & 20.9 & 1.55 & \\
& L2 & 153 & 1.55 & \\
& L3 & 251 & 1.55 & \\
& M2 & 0.8 (from ratio to M4) & 1.454 & 1.454 \\
\hline
\end{tabular}

This research was founded by the common EFRE program of the European Commission and the country Berlin under contract number 10127509.

References

[1] A. Bjeoumikhov et al., X-ray spectrom. 34 (2005), 493.

[2] B.J. Cross \& K.C. Witherspoon, Microscopy Today 12 (2004), 20.

[3] X-ray tube MCMB50, Roentgentechnik Warrikhoff, D-xxxx Neuenhagen, Germany

[4] F. Scholze \& M. Procop, X-ray Spectrom 30 (2001), 69.

[5] M. Procop, Microsc. Microanal. 10 (2004), 481.

[6] M. Alvisi et al., Book of Abstracts of $9^{\text {th }}$ European Workshop on Modern Developments and Applications in Microbeam Analysis, Florence, Italy, 2005, 189 (submitted to Microsc. Microanal.).

[7] J.L. Pouchou \& F. Pichoir, Electron Probe Quantitation, Plenum Press 1991, p.31.

[8] D.C. Joy, J.Microscopy 191, Pt.1 (1998), 74.

[9] W.T. Elam et al. Radiation Phys. Chem. 63 (2001), 121.

[10] J.L. Pouchou et al., Proceedings XII ICXOM, Krakow 1989, 52. 